Miller, J., Zhu, H. and Zhang, J. (2017) 'Guest Editorial: Advances in Web Services Research', IEEE Transactions on Services Computing, 10 (1), pp. 5-8.

DOI: https://doi.org/10.1109/TSC.2016.2619518

This document is the authors' Accepted Manuscript.

License: https://creativecommons.org/licenses/by-nc-nd/4.0

Available from RADAR: https://radar.brookes.ac.uk/radar/items/8fec83ea-05dc-4474-9666-3a5d2f4c79b3/1/

Copyright $(\subseteq$ and Moral Rights are retained by the author(s) and/ or other copyright owners unless otherwise waved in a license stated or linked to above. A copy can be downloaded for personal non-commercial research or study, without prior permission or charge. This item cannot be reproduced or quoted extensively from without first obtaining permission in writing from the copyright holder(s). The content must not be changed in any way or sold commercially in any format or medium without the formal permission of the copyright holders. 


\title{
Guest Editorial: Advances in Web Services Research
}

John A. Miller, Member, IEEE, Hong Zhu, Senior Member, IEEE, and Jia Zhang, Member, IEEE

\begin{abstract}
Research on Web Services and Services Computing began shortly after the start of the new millennium and with the first major research conference on the subject, the International Conference on Web Services, starting in 2003. Due to the new and practical utility of Web service technology, many researchers began to work in this field. New ideas, applications and related technologies continue to invigorate this discipline. This special issue of IEEE Transactions on Services Computing considers four such topics that lead to "Advances in Web Services Research": Cloud Services; Polices and Agreements; Services Engineering; and Service/Process Mining. It consists of the best extended papers from the two premier research conferences on services computing: the 2015 IEEE International Conference on Web Services and the 2015 IEEE Conference on Services Computing.
\end{abstract}

Index Terms-Web services, services computing, cloud services, policies and agreements, services engineering, service/process mining

\section{Introduction}

Research in Web Services and Services Computing has progressed tremendously in the last decade, yet remains very active and highly engaging. Research in this field has had large positive impacts on industrial practice in multi-billion dollar sectors of the economy. Services are everywhere. Continued progress in research and development in the areas of Web Services and Services Computing can spur further productivity and even greater usage.

The IEEE Transactions on Services Computing (TSC) has sponsored a special issue on Advances in Web Services Research that consists of extended versions of the best and most innovative papers from the 2015 IEEE International Conference on Web Services [1] and the 2015 IEEE Services Computing Conference [2].

The IEEE International Conference on Web Services (ICWS) has been the prime international forum for both researchers and industrial practitioners to exchange the latest advances in the state of the art and practice of Web Services, to identify emerging research topics, and to define the future of Webbased services. After merging with the IEEE
European Conference on Web Services (ECOWS) in 2012, in 2015 ICWS celebrated its 22nd event in New York, NY, USA.

The IEEE Services Computing Conference (SCC) as a sister conference of ICWS takes a slightly broader view of Services Computing, considering business modeling, business consulting, solution creation, service delivery, and soft-ware architecture design, development and deployment. In 2015, SCC celebrated its 12th event, co-located with ICWS 2015, in New York, NY, USA.

This special issue received 71 extended papers of which 10 were selected, based on a thorough review process, for publication (14 percent acceptance rate). These papers focus on the following four topics: Cloud Services, Policies and Agreements, Services Engineering, and Service/Process Mining. In the sections below, each topic is introduced and includes references to papers that provide additional back-ground/orientation to the topic.

\section{Cloud Services}

Although the term Cloud Services can have different meanings, this special issue mainly focuses on where Cloud Computing (CC) meets the Service- 
Oriented Architecture (SOA), as described in [3], [4]. For example, Software as a Service (SaaS), which in a full cloud stack [5] resides above Infra-structure as a Service (laaS) and Platform as a Service (PaaS), is a natural hosting location for Cloud services. Advantages of using cloud computing to host services include resource sharing, protections offered by virtualization and state-of-the-art security, elasticity to handle changing demand for services, and managed upgrades, as well as higher availability and reliability.

In the case of multiple tenants providing composite Cloud services within a single SaaS instance, it is important to optimize overall performance for the tenants by maximizing sharing of services/resources and appropriately managing service executions. In the paper titled, "Software-Defined Service Networking: Performance Differentiation in Shared Multi-Tenant Cloud Applications," Indika Kumara, Jun Han, Alan Colman, and Malinda Kapuruge argue that the design and execution of Cloud services can be more effective if based on the concept of service networks rather than conventional process-oriented designs, e.g., utilizing BPEL or BPMN. Under the service network approach, individual tenants utilize a subset of the services/resources in the network to execute their processes/composite services. This approach can result in greater sharing, improved through-put and reduced response times.

Formal approaches for assuring correct operation of composite Web services/business processes are gaining ground due to advances in formal specification languages (e.g., Event-B), theorem provers and model checkers, as well as packages such as Rodin. This is particularly true for business processes running in the Cloud. In the paper titled, "Towards Correct Cloud Resource Allocation in Business Processes," Mohamed Graiet, Amel
Mammar, Souha Boubaker, and Walid Gaaloul utilize Event-B and Rodin for developing processes that will satisfy a set of invariants to help ensure correctness of the business pro-cess. The paper goes through several steps, but focuses on defining Cloud resources at the laas level and how they are allocated and shared by business processes. Shareability and elasticity characteristics of Cloud resources are formally specified.

While the previous paper examined correctness of Cloud resource allocation at the laas level, the next paper examines the development of markets to trade laaS level Cloud resources. Due to the heterogeneous and complex nature of Cloud infrastructure, efficient open market mechanisms have not progressed sufficiently to be put into widescale practice. In the paper titled, "Towards Efficient and Truth-ful Market Mechanisms for Double-sided Cloud Markets," Sergei Chichin, Quoc Bao Vo, and Ryszard Kowalczyk develop mechanisms for double-sided markets with multi-ple buyers (bids) and sellers (asks) of heterogeneous Cloud resources. Resources are allocated to maximize the satisfaction of both buyers and sellers and formulated as a Multi-Dimensional Knapsack Problem (MMKP). As MMKP is NP-hard, greedy heuristics are applied and compared with optimal solutions provided by an Integer Linear Programming (ILP) solver. The greedy heuristics run much faster and often produce near-optimal solutions.

\section{Policies and Agreements}

As the services economy continues to expand, the needs for improved service management [6] in general and detailed policies and agreements in particular, have expanded as well. Thus, there are increasing needs for more comprehensive agreement specification languages as well as the ability to check the validity of the specifications. 
Along these same lines, it is becoming critical to guarantee certain levels of privacy and security in service interactions.

A Service Level Agreement (SLA) can be used by business entities in order to establish business arrangements. In order to speed up and facilitate this process, automation based on standardized and/or ontology backed specifications are essential. In the paper titled, "Modeling Service Level Agreements with Linked USDL Agreement," Jose Maria Garcia, Pablo Fernandez, Carlos Pedrinaci, Manuel Resinas, Jorge Cardoso, and Antonio RuizCortes introduce Linked USDL Agreement into the family of Linked Unified Service Description Language (USDL) specifications and ontologies. Linked USDL Agreement allows for comprehensive specification of service agreements, including technical, business and legal aspects, and has tool support providing validity checking to help users correctly specify agreements. The tool support also provides a mapping to Open Grid Forum's WSAgreement technically-oriented specification.

Just like the rest of computing technology, security and privacy issues are becoming increasingly more important for services computing. Certain information (e.g., a trade secret) that might ordinarily be found in a Web service composition/process is said to be opaque if it cannot be deduced by observing the execution of the services. In the paper titled, "On the Verification of Opacity in Web Services and their Composition," Amina Bourouis, Kais Klai, Nejib Ben Hadj-Alouane, and Yamen El Touati provide a formal technique to ensure that certain information can be verified to be opaque.

\section{Services Engineering}

Research on services engineering [7] addresses the problems raised in the development, maintenance and operation of service-oriented systems. It covers a wide range of software engineering areas, including design, testing, debugging, run-time monitoring, and verification and validation, etc. It has been an active research topic for the past several years. This special issue contains three papers on services engineering: (1) finding data races in multithreaded service environments, (2) localizing runtime anomalies, and (3) proving the correctness of compositions that include compensation.

Modern multi-threaded execution of services may results in data races, involving threads accessing the same location in an uncontrolled manner. In the paper titled "SDA-CLOUD: A Multi-VM Architecture for Adaptive Dynamic Data Race Detection," Chunbai Yang, W. K. Chan, Chang-jiang Jia, and Yuen Tak $\mathrm{Yu}$ present a testing technique for detecting data races in Web service programs. They employ multiple virtual machines running multiple copies of the same service components to improve the detection rate of data races. Their experiment reveals that the approach is feasible in monitoring multiple compiled versions. By taking existing race detectors as building blocks, it can improve race detection ability by detecting various types of races. Given a limited execution budget, the approach is also significantly more effective than approaches that use single compiled versions only.

Service-oriented systems should try to maintain the Quality-of-Service (QoS) of the component services that they manage/execute. While detection of runtime anomalies that degrade QoS is needed, the overhead of full run-time monitoring is often too costly. In the paper titled "Localizing Runtime Anomalies in Service-Oriented Systems," Qiang He, Xiaoyuan Xie, Yanchun Wang, Dayong Ye, Feifei Chen, Hai Jin, and Yun Yang present a spectrumbased approach for limited, yet focused run-time monitoring of service-oriented systems to quickly localize run-time anomalies. Their extensive 
experiments compare three variations of the their delay based approach with both random inspection and Ochiai-based inspection for localizing single and multiple run-time anomalies. The findings indicate that their approach represents a significantly improvement over the other approaches.

Modern languages for composing/orchestrating Web services (e.g., BPEL) provide features to support compensation in the face of failures. Unfortunately, the correctness of the compensation has been left up to the designer/developer. Formally proving correctness of compensation down to the functional level is very challenging. In the paper titled "Web Services Compensation at Runtime: Formal Modeling and Verification Using the Event-B Refinement and Proof Based Formal Method," Guillaume Babin, Yamine Ait-Ameur, and Marc Pantel employ Event-B, a formal method, to address the problem of run-time service compensation. Based upon invariants, platforms such as Rodin can be used to prove correctness of compensation actions. For equivalent compensation, it must complete the functional objective of the service, while safely preserving the steps completed by the service. The authors illustrate how their approach works via a detailed case study.

\section{Service/Process mining}

Recently, there has been greatly increased research activity in service/process mining [8]. Business processes, work-flows and Web service compositions are becoming integral to the operations of modern corporations. When change is needed because of, for example, merger, acquisition, new products or new services, processes need to merged, re-engineered or newly created. Given the increasing size of model repositories, it is now necessary to have tools for searching, browsing, querying or mining (including. sophisticated matching techniques) for appropriate business pro-cess models. Furthermore, information about the dynamics of processes is typically contained in event logs. Sophisticated mining techniques are needed to extract useful information from process event logs.

In many cases, one wishes to find business process models that are similar, with many services/tasks in common as well as possibly similar control and data flow. In the paper titled, "Querying Similar Process Models based on the Hungarian Algorithm," Bin Cao, Jiaxing Wang, Jing Fan, Jianwei Yin, and Tianyang Dong provide a means for finding similar business process models. The processes are modeled as Petri nets from which similarity metrics based on transitions and places (the two types of nodes in Petri nets) are developed. Using these metrics and the Hungarian algorithm (optimal matching in bipartite graphs), similar business process models are effectively and efficiently found.

Information about the execution of business processes is typically contained in event logs. This information can be used for correcting implementations, process enhancement, performance evaluation and conformance checking. To fully utilize the large amount of information contained in event logs, it is important to align event logs with process models. Unfortunately, this problem has been shown to be NP-hard [9]. In the paper titled, "Efficient Alignment between Event Logs and Process Models," Wei Song, Xiaoxu Xia, Hans-Arno Jacobsen, Pengcheng Zhang, and Hao $\mathrm{Hu}$ develop an effective and efficient heuristic algorithm to align event logs with business processes modeled as Petri nets. A thorough evaluation, shows that their algorithm often produces better alignments and runs much faster than the current state-of-the-art approaches. 


\section{Summary}

This special issue of the IEEE Transactions on Services Computing has presented state-of-the-art research in four distinct areas of services computing.

First, the synergy provided by Services Computing and Cloud Computing has potential for improving both areas. Indeed the strong connection between them has been fore-seen by the Services Society, as the IEEE International Conference on Cloud Computing has been a sister conference to ICWS and SCC since 2010.

Second, due to the rapid uptake of services computing by business and industry, the need for comprehensive polices and service agreements covering technical, business and legal aspects has led to important and useful work in this area.

Third, it is observed that the research area of Web services has been significantly widened. It now covers a broad spectrum of topics that range from social aspects as mention above, to the enabling techniques and infrastructure, and to services engineering. The sub-field of services engineering includes development, testing, operation, and maintenance of service-oriented systems.

Finally, due to the practical needs of businesses for finding, using and improving their workflows, processes and service compositions, research on service/process mining has expanded rapidly, and includes work on process matching and event log extraction

\section{Acknowledgements}

The guest editors would like to thank all of the reviewers for their essential contributions and hard work. Special thanks goes to the EiC, Ling Liu and the Special Issues Editor, Rong N. Chang for their invaluable guidance and support throughout the process.

\section{References}

[1] J. A. Miller and H. Zhu, Eds., Proc. 22nd IEEE Int. Conf. Web Serv., Jun./Jul. 2015.

[2] P. P. Maglio, I. Paik, and W. Chou, Eds., Proc. 12th IEEE Conf. Serv. Comput., Jun./Jul. 2015.

[3] L.-J. Zhang and Q. Zhou, "CCOA: Cloud computing open architecture," in Proc. IEEE Int. Conf. Web Serv., 2009, pp. 607-616.

[4] K. He, A. Fisher, L. Wang, A. Gember, A. Akella, and T. Ristenpart, "Next stop, the cloud: Understanding modern web service deploy-ment in EC2 and Azure," in Proc. Conf. Internet Measurement Conf., 2013, pp. 177-190.

[5] I. Bojanova and A. Samba, "Analysis of cloud computing delivery architecture models," in Proc. IEEE Workshops Int. Conf. Adv. Inf. Netw. Appl., 2011, pp. 453-458.

[6] M. P. Papazoglou and W.-J. Van den Heuvel, "Web services man-agement: A survey," IEEE Internet Comput., vol. 9, no. 6, pp. 58-64, Nov./Dec. 2005.

[7] L.-J. Zhang, H. Cai, and J. Zhang, Services Computing. Berlin, Germany: Springer, 2007.

[8] W. van der Aalst, "Service mining: Using process mining to dis-cover, check, and improve service behavior," IEEE Trans. Serv. Comput., vol. 6, no. 4, pp. 525-535, Oct./Dec. 2013.

[9] J. Wang, S. Song, X. Zhu, and X. Lin, "Efficient recovery of missing events," Proc. VLDB Endowment, vol. 6, no. 10, pp. 841-852, 2013. 